\title{
ANÁLISIS 3D MEDIANTE ELEMENTOS FINITOS DEL DISEÑO DEL SISTEMA DE FIJACIÓN DE PLACAS CRANEALES
}

\section{D FINITE ELEMENT ANALYSIS OF THE DESIGN OF SYSTEM FIXING CRANIAL PLATES}

\author{
Rodrigo López*, Sergio Carvajal* \\ ${ }^{*}$ Universidad Santo Tomas, Bogota,5878797, Colombia \\ Email: oscarlopez@usantotomas.edu.co. \\ sergiocarvajal@usantotomas.edu.co.
}

\begin{abstract}
Resumen: El traumatismo craneal es causado por accidentes de tránsito, caídas, agresiones o en prácticas deportivas, según la Organización Mundial de la Salud (OMS) este traumatismo es bastante común a nivel Mundial y significativo en Colombia, afectando a gran parte de la población, principalmente a personas menores de 45 años. El objetivo de este trabajo fue el de establecer una metodología que permitiera realizar el diseño personalizado de placas craneales, enfocándose en la optimización del mecanismo de anclaje de la placa al hueso que compone el cráneo. Para esto, el dominio de trabajo (cráneo) con la afectación craneal fue reconstruido a partir de tomografías computacionales, el diseño de la placa fue realizado mediante el uso de software CAD y mediante el uso de elementos finitos se determinó el comportamiento biomecánico del conjunto implantado, determinando el efecto mediante la aplicación de cargas de impacto del sistema de sujeción de la placa al tejido óseo.
\end{abstract}

Palabras clave: Cráneo, Tomografía, Elementos finitos, Biomecánica.

\begin{abstract}
The cranial traumatism is caused by accidents of traffic, falls, aggressions or in sports practices, according to the World Health Organization (WHO) this traumatism is common enough worldwide and significantly in Colombia, concerning great part of the population, principally to 45-year-old minor persons. The aim of this work was of establishing a methodology that was allowing to realize the personalized design of cranial plates, focusing in the optimization of the mechanism of anchorage of the plate on the bone that composes the cranium. For this, the domain of work (cranium) with the cranial affectation was reconstructed from tomography computational and the design of the plate was realized by means of the use of software CAD and by means of the use of finite elements the biomechanical behavior decided of the well-established set, determining the effect by means of the application of loads of impact of the system of subordination of the plate to the bony tissue.
\end{abstract}

Keywords: Skull, Tomography, Finite Elements, Biomechanics.

\section{INTRODUCCIÓN}

El traumatismo craneal es comúnmente causado por accidentes de tránsito, caídas, agresiones o en prácticas deportivas, según la Organización Mundial de la Salud (OMS), este traumatismo craneal es a nivel Mundial es una de las causas de muerte más significativas, afectando a gran parte de la población, principalmente a personas menores de 45 años según(Ortiz 2006). Jennett B. Manifiesta que es comúnmente conocido como la "epidemia silenciosa" por sus altos índices de mortalidad y discapacidad teniendo un índice de incidencia de 200 a 400 por 100.000 habitantes(Domínguez Peña, Hodelín Tablada, and Antonio Fernández Aparicio 2010), de modo que es de utilidad el uso de métodos 
ingenieriles que lleven a la reconstrucción de placas craneales de forma rápida y eficaz, con aplicación de material con alta biocompatibilidad como lo es el titanio y sus aleaciones(Comercia and Pijho n.d.), por tal razón, se busca establecer una metodología que permita realizar el diseño personalizado de placas craneales, enfocándose en el mecanismo de anclaje de la placa al tejido óseo presente en el cráneo.

Para esto el dominio de trabajo (cráneo) con la afectación craneal en hueso frontal, es reconstruido a partir de tomografías computacionales, el diseño de la placa es realizado mediante el uso de software $\mathrm{CAD}$, y utilizando el método de elementos finitos (MEF), donde encontramos aproximaciones de orden continuo a los problemas con el fin de establecer un numero finito de elementos, los cuales forman un mallado superficial en las piezas siguiendo la región geométrica y las aristas del mallado son conocidas como nodos que se encargan de definir el comportamiento de las funciones que describen el análisis computacional en el software ANSYS.

Por ende este método va a permitir transformar un cuerpo de naturaleza continua en un modelo discreto aproximado(Durango 1995), donde se evalúan los efectos mediante la aplicación de cargas de impacto en el sistema de sujeción de la placa al tejido óseo; esto llega a permitir que pacientes que han sufrido traumatismos craneales puedan tener una mayor probabilidad de vida después de sus accidentes y que la afectación dentro de su sistema corporal sea menor a otros que no cuentan con estos sistemas.

\section{METODOLOGIA}

\subsection{Reconstrucción craneal}

\subsubsection{Estudio anatómico}

Para la reconstrucción craneal se hizo un estudio anatómico del cráneo (Torres et al. 2014) donde se determinaron los huesos principales y sus componentes funcionales.

Los huesos craneales están formados anatómicamente por hueso cortical (duro) y hueso trabecular (blando), la zona afectada es el hueso frontal como se muestra en la (figura 1), considerado como hueso plano en la anatomía humana y conforma la parte de la frente hasta debajo de las cejas, el cual tiene simetría al plano sagital del cuerpo humano y cumple la función de proteger el encéfalo y los órganos de audición.

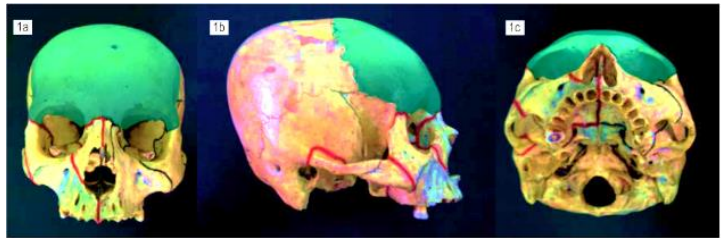

Fig. 1. Vista de los diferentes planos del hueso frontal en la sección 1a, 1b, y 1c para identificar la zona de trabajo.(Avello-Canisto, Avello-Peragallo 2008)

\subsubsection{Reconstrucción a partir de tomografías computacionales}

El procedimiento para realizar el diseño y análisis de los tipos de mecanismos de sujeción de placas craneales consistió en una primera etapa en la reconstrucción mediante el uso de tomografías computacionales de un cráneo con una afectación severa en el hueso frontal, como se observa en la (figura 2). Durante el proceso de reconstrucción fue necesario depurar la geometría del cráneo eliminando superficies que no harían parte de la zona afectada por la lesión, como se observa en la figura 3. Luego de eliminar las zonas no necesarias se suavizaron las superficies del cráneo para prepararlas y realizar el diseño de la placa craneal (ver figura 3).

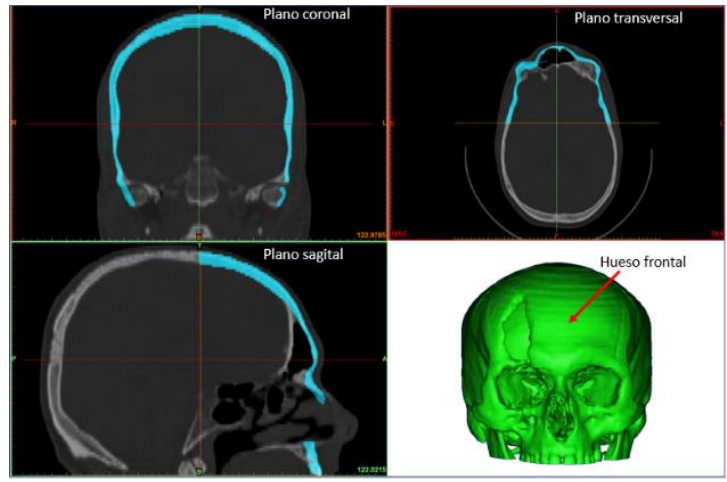

Fig. 2. Tomografías cráneo y afectación hueso frontal

\subsection{Diseño y modelado de la placa craneal}

Para realizar el diseño de la placa se necesita obtener la curva característica del cráneo, en la zona afectada con el fin de que la placa tenga el mismo perfil geométrico de un cráneo sin afectación y minimizar los problemas causados por el traumatismo. 

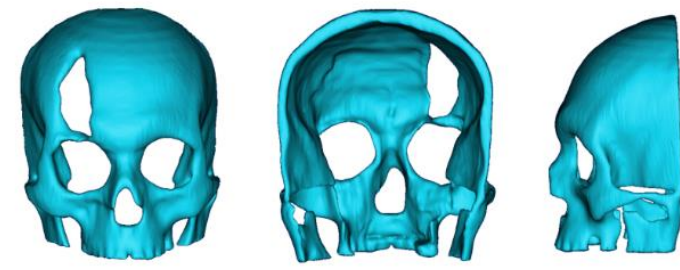

Fig. 3. Cráneo con acabado superficial

Para este fin se tiene la zona de trabajo (cráneo con afectación) en óptimas condiciones para minimizar posibles errores por material sobrante o excesivo, teniendo suavizado el cráneo (figura 3 ) y preparado el contorno de la placa (figura 4), se procede a delimitar la zona afectada y se crea una curva que se adapte a la fractura craneal formando así el contorno por donde va a ser generada la placa.
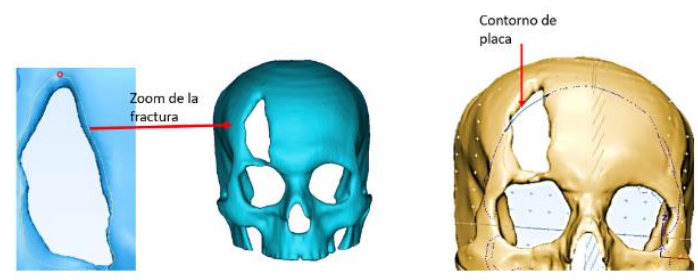

Fig. 4. Contorno zona afectada

Teniendo todos los parámetros definidos se inicia la reconstrucción de la placa en software CAD, modelando la geometría que mejor se adapte a la zona de afectación para lograr un mejor comportamiento entre la placa y el hueso frontal.

En la reconstrucción se tiene en cuenta la edad del paciente ya que es un factor fundamental, ya que, dependiendo de esta variable se sabe en qué etapa de desarrollo se encuentra el cráneo, en este caso no se tenía información del paciente, por este motivo se hicieron estudios cefalométricos(Ramires et al. 2011), para definir las medidas antropométricas en las que se encuentra este caso específico y sé obtiene que es un hueso ya maduro, que puede oscilar entre los 20 y 45 años de edad, al cual ya se le puede realizar un sistema de sujeción adecuado, caso que no ocurre para los huesos de personas menores de 18 años, ya que se encuentran en desarrollo, lo que anexa variables no previsibles en su totalidad en sus medidas y características como material al pasar del tiempo.
Teniendo ya estas consideraciones se dispone a realizar el diseño y construcción de la placa (figura 5), en donde se considera la geometría de la lesión para el diseño apropiado del sistema placa - hueso frontal y realizar la mejor alternativa para este caso personalizado.
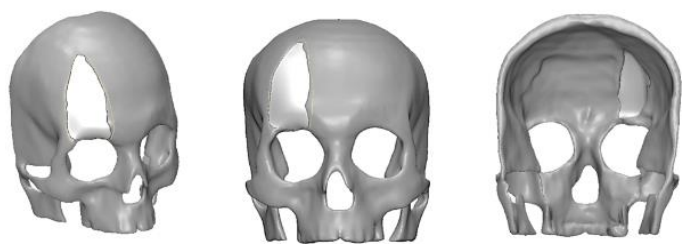

Fig. 5 Diseño placa

Una vez diseñado el implante con unas condiciones similares a las de un cráneo sin afectación, se inicia el análisis de los diferentes métodos de sujeción(DePuy Synthes. 2016), que se pueden adaptar y estudiar su comportamiento óptimo en nuestro sistema, inicialmente con la placa preliminar (figura 6) además se ve la posibilidad de implementar piel artificial en la zona de reconstrucción para una mejor evolución y adaptación al paciente (Esp. Hans C. Ramos López, MSc. Antonio Gan Acosta 2007).
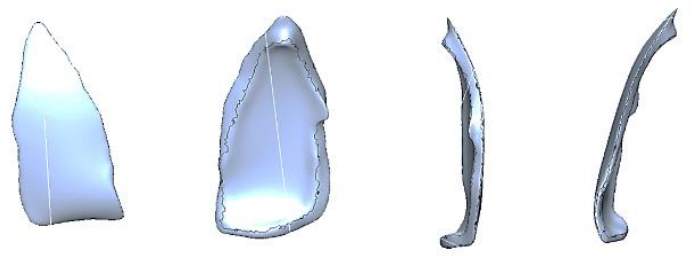

Fig. 6. Placa craneal

Para la obtención de datos iniciales y evaluación del comportamiento, se trabajó con la placa basándose en el método de sujeción osteointegración (proceso biológico de integración del implante y el hueso), teniendo como base la placa preliminar (figura 6).

\subsection{Determinación del comportamiento biomecánico en ANSYS}

\subsubsection{Modelo de elementos finitos}

Al diseño geométrico de la placa fue necesario realizar un suavizado en su perfil para lograr un contacto perfecto entre la placa y el hueso frontal, ya que si no ocurre puede causar problemas en la solución por medio de elementos finitos, 
presentando conflicto en los resultados, por esto, se debe garantizar que son dos elementos diferentes y se toman como una unión donde se definirá la zona de contacto o frontera de operación (figura 8).

Posteriormente ya con la placa y el cráneo en buenas condiciones para operación de análisis por elementos finitos en el software ANSYS se realiza la importación del conjunto a la interfase de Workbench (figura 7), donde se proceda a la discretización de la placa y el cráneo, con el fin de evaluar los efectos causados por cargas de impacto al sistema.

Para el análisis se realiza la determinación del comportamiento biomecánico entre la placa y el hueso frontal, donde se trabaja en el módulo estructural de Workbench ANSYS 19.1®. Para el análisis del sistema es necesario considerar las propiedades de los materiales, la geometría del modelo, las condiciones de frontera, las cargas y el enmallado de los diferentes sólidos.

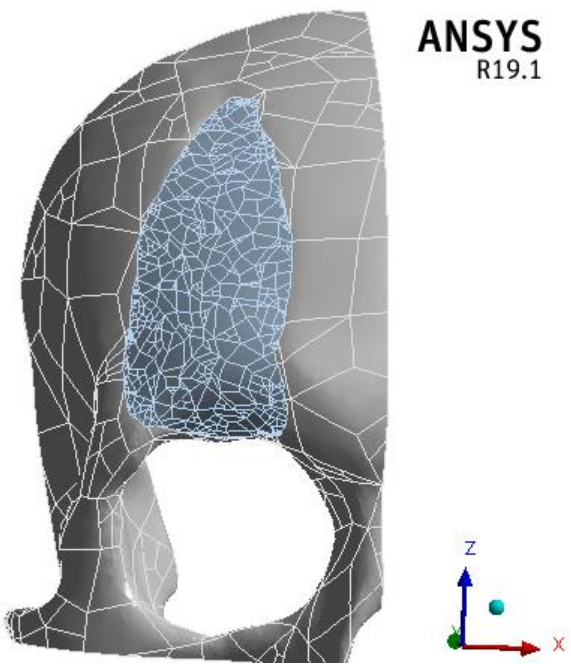

Fig. 7. Geometría craneal cargada en ANSYS Workbench módulo estructural.

\subsubsection{Propiedades de los materiales}

Las propiedades de los materiales son factores indispensables en la solucion de problemas de elementos finitos, ya que definen el comportamiento elastico, plastico, ductil, fragil, entre otras propiedades mecanicas, con el fin de describir el comportamiento estructural y simular lo que ocurre en la realidad con la aplicación de cargas dinamicas y estaticas.
En el proceso de solucion mediante software ANSYS es necesario cargar los materiales a el modulo engeneering data para generar los componentes que definen el comportamiento y obtener los resultados que se asemejan a la realidad, para esto se buscan las propiedades mecanicas del hueso frontal y del titanio puro, las cuales muestran los factores relevantes (tabla 1 y tabla 2 ).

\begin{tabular}{|l|l|l|}
\hline Propiedades & Valor & Unidades \\
\hline Densidad & 1800 & $\mathrm{~kg} / \mathrm{m}^{3}$ \\
\hline $\begin{array}{l}\text { Módulo de } \\
\text { Young's }\end{array}$ & 12500 & $\mathrm{Mpa}$ \\
\hline $\begin{array}{l}\text { Coeficiente } \\
\text { de Poison }\end{array}$ & 0,23 & \\
\hline \multicolumn{2}{|c|}{$\begin{array}{l}\text { Tabla 1. Propiedades mecánicas hueso } \\
\text { frontal(Gonzales., M; Isanza. 2016) }\end{array}$}
\end{tabular}

\begin{tabular}{|l|l|l|}
\hline Propiedades & Valor & Unidades \\
\hline Densidad & 6420 & $\mathrm{~kg} / \mathrm{m}^{3}$ \\
\hline $\begin{array}{l}\text { Módulo de } \\
\text { Young's }\end{array}$ & 96000 & $\mathrm{Mpa}$ \\
\hline $\begin{array}{l}\text { Coeficiente } \\
\text { de Poison }\end{array}$ & 0,36 & \\
\hline
\end{tabular}

Tabla 2. Propiedades mecánicas titanio(Pérez Pozo et al. 2015)

\subsubsection{Condiciones de frontera, restricciones y cargas}

Continuando con el esquema del proyecto, se generan las condiciones de frontera, para tal fin se realizó un contacto entre la placa y el hueso frontal de tipo Bonded (Analysis 2012) (simulando el proceso de osteointegración entre estos dos componentes) (figura 8).

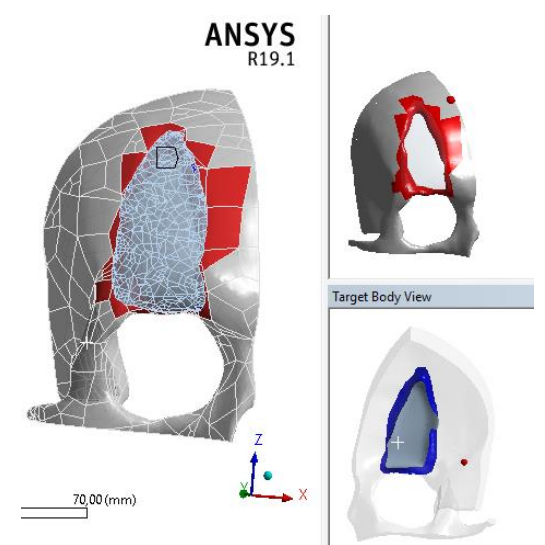

Fig. 8. Generación de contacto entre placa y hueso frontal. 
Teniendo en cuenta las restricciones se fija el cráneo para que la carga aplicada, tenga un movimiento similar al de un cráneo que sufre una lesión severa por impacto en la placa, para esto se toman las regiones donde se realizaron los cortes en el plano sagital, coronal y transversal para tomarlos como soportes fijos.

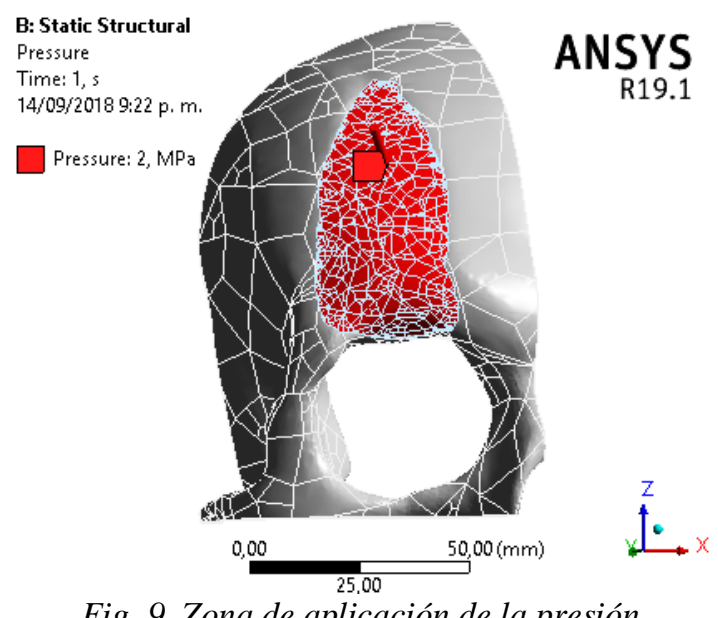

Fig. 9. Zona de aplicación de la presión

Para la presión aplicada (Edgar and Ramírez 2017) (figura 9) se determinó toda la superficie posterior de la placa, donde se analizó con el fin de observar el comportamiento del conjunto placa y tejido óseo frontal, para adecuar los diferentes tipos de sujeción, observar cual es el método óptimo para fijación de la placa, mejorando el sistema y reduciendo los sobre esfuerzos al cráneo.

\subsubsection{Generación del mallado}

Se realiza una operación de simetría en el cráneo recortando la geometría a la mitad, para disminuir el número de elementos a analizar y con esto ganar tiempo en la obtención de resultados, teniendo 352,110 nodos y 234,774 elementos en la malla generada (figura 10), con una calidad media y un tamaño grueso, el cual para la primera etapa se obtiene bastante información, que puede ser utilizada en análisis posteriores.

El mallado es un parámetro indispensable en la aplicación de este tipo de simulaciones, ya que por medio de este se genera una superficie ambigua a la geometría del cráneo y la placa, definiendo el comportamiento del sistema, recolectando información en cada nodo, para que la obtención de los resultados sea precisa.

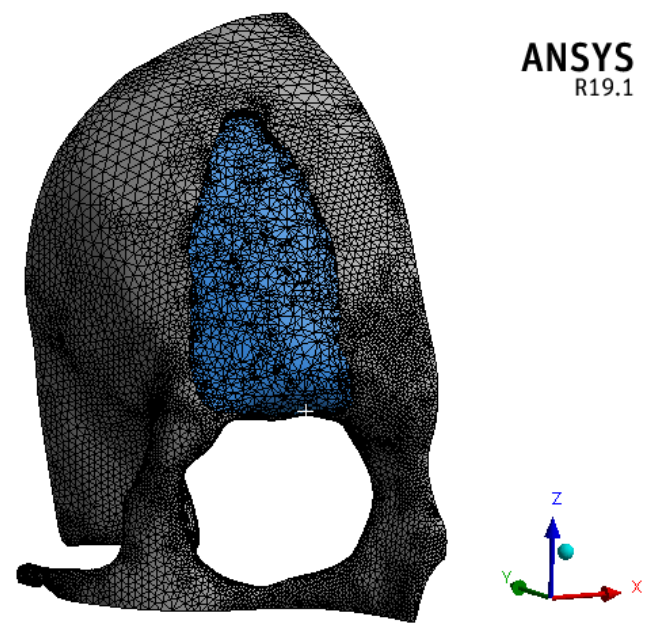

Fig. 10. Mallado

\section{RESULTADOS}

\subsection{Esfuerzos en el cráneo y la placa}

Como resultado del análisis realizado se obtuvo la distribución de esfuerzos y deformaciones del conjunto placa, cráneo. Con el fin de obtener el máximo valor de esfuerzos en el sistema y observar los puntos o zonas críticas, teniendo como resultado de máximo esfuerzo de 103,62 Mpa (figura 11), y se evidencia en la zona de color rojizo (zonas críticas) y color azul a zonas sin mayor afectación.

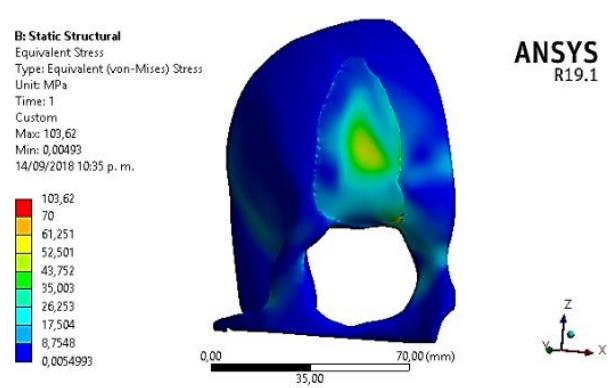

Fig. 11. Esfuerzo máximo y mínimo

Posteriormente, se observa que ocurre en cada componente por separado (figura 12 y 13), para poder determinar qué zona es crítica en el hueso frontal y en la placa. 


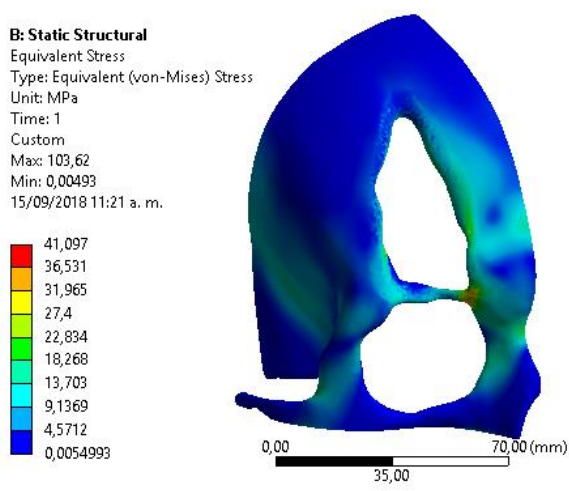

Fig. 12. Esfuerzos en cráneo

Analizando los esfuerzos máximos generados en el cráneo (figura 12), se puede identificar que no son críticos con un valor de 41,1 Mpa, por ende, da a entender que los esfuerzos máximos están en la placa (figura 13) y allí es donde se tiene que elaborar un diseño de sujeción que contribuya a minimizar los sobre esfuerzos.

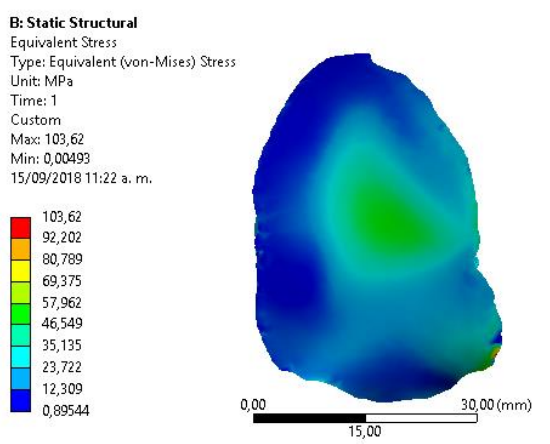

ANSYS

Fig. 13. Esfuerzos en placa

\subsection{Deformaciones en el cráneo y la placa}

Las deformaciones en el conjunto se presentan en la zona rojiza (figura 14), donde se habla en el orden de centésimas de milímetro de desplazamiento ocasionado, lo que quiere decir que con esta carga aplicada el efecto causado es mínimo tanto en la placa como en el cráneo ya que no supera el límite elástico del material.
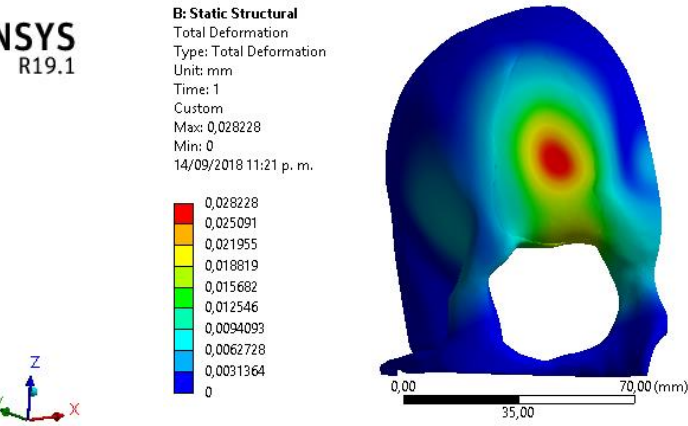

ANSYS
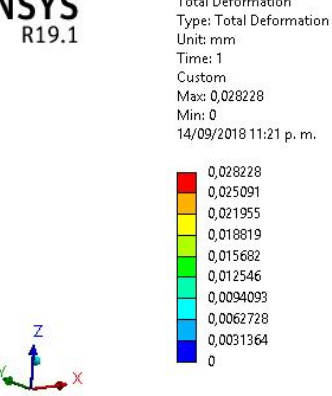

Fig. 14. Deformaciones

La mayor elongación presentada es de 0,028 mm en el conjunto hueso frontal, placa (figura 14).

\section{CONCLUSIONES}

Con el presente artículo se muestran los resultados iniciales para un análisis mediante elementos finitos a el conjunto (placa, hueso frontal) donde se determina que lo mejor para este tipo de geometría es realizar un suavizado óptimo para evitar la interferencia en la obtención de resultados, además teniendo como base los esfuerzos máximos o de Von mises para criterios de falla, se determina que el material se está comportando de una manera adecuada en el conjunto y que el diseño preliminar está listo para iniciar la elaboración de los tipos de anclaje de la placa al cráneo.

\section{RECONOCIMIENTO}

Este trabajo se realizó con recursos de la decimosegunda convocatoria interna para el fomento de la investigación FODEIN-2018 de la Universidad Santo Tomás.

\section{REFERENCIAS}

Analysis, State Thermal. 2012. "Introduction to ANSYS Mechanical.” Release 15.0: 1-14.

Avello-Canisto, Francisco, and Allan AvelloPeragallo. 2008. "Nueva Clasificación de Las Fracturas Del Tercio Superior Facial: Consideraciones Anatomo-Quirúrgicas." Anales de la Facultad de Medicina 69(4): 272-77.

Comercia, E L Titanio, and Mknte Pijho. "Aplicaciones Biomédicas Del Titanio v Sus Aleaciones." E.T.S. ingenieros industriales bacelona (c): 34-42.

DePuy Synthes. 2016. "MatrixNEURO. El Sistema de Placas Craneales de La Próxima Generación." división de Synthes GmbH.

Domínguez Peña, Rafael, Ricardo Hodelín 
Tablada, and Marco Antonio Fernández Aparicio. 2010. "Factores Pronósticos En El Traumatismo Craneoencefálico Grave. Prognoses Factor in Serious CranioEncephalic Traumatism." Mediciego 16(2).

Durango, Néstor. 1995. "Condicion de Frontera En El Metodo de Los Elementos Finitos." Ingenieria y desarrollo 1: 24-28.

Edgar, M C, and Isaac Ramírez. 2017. "Modelado de Fractura Del Cráneo Bajo Condiciones de Impacto."

Esp. Hans C. Ramos López1, MSc. Antonio Gan Acosta2, MSc. Jorge L. Díaz R.2. 2007. "PIEL ARTIFICIAL." Revista Colombiana de Tecnologías de Avanzada 2(March): 11116.

Gonzales., M; Isanza., J.; 2016. "Desarrollo de Un Modelo FEM Del Complejo Craneofacial Para Simular Tratamientos." CES Odontología Vol. 23-(January 2010).

Ortiz, Armando. 2006. "Traumatismo Encefalocraneano (TEC). Una Puesta Al Día." Academic Emergency Medicine 17(3): 98-105. http://doi.wiley.com/10.1111/j.13600443.1995.tb02822.x.

Pérez Pozo, Luis, Fernando Briones Picheira, Sheila Lascano Farak, and Claudio Aguilar Ramírez. 2015. “Análisis de Esfuerzos Mediante El Método de Elementos Finitos de Implantes Dentales de Titanio Poroso." Stress analysis of porous titanium dental implants by using the finite element method. 33(1): 80-97.

Ramires, Rossana Ribeiro et al. 2011. "Medidas Faciais Antropométricas de Adultos Segundo Tipo Facial e Sexo.” Revista CEFAC 13(2): 245-52.

Torres, Ing Germán, Hernando Velandia, Carlos Álvarez, and Luis Enrique Mendoza. 2014. "Diseño e Implementación de Un Software, Aplicado Al Estudio y Análisis de La Estética Facial En Ortodoncia." Revista Colombiana de Tecnologías de Avanzada 2(24): 1-8. 